\title{
Determination of the position and vibrational amplitude of an adsorbate by means of multiple-order $\mathrm{x}$-ray standing-wave measurements
}

\author{
M. J. Bedzyk* and G. Materlik \\ Hamburger Synchrotronstrahlungslabor at Deutsches Elektronen-Synchrotron, D-2000 Hamburg 52, Germany
}

(Received 10 January 1985)

For a submonolayer of $\mathrm{Br}$ atoms adsorbed onto a real $\mathrm{Ge}(111)$ surface, $\mathrm{x}$-ray interference fields were used to determine the $\mathrm{Br}$ position with respect to the substrate (111) and (333) diffraction planes. The results from both the (111) and (333) measurements are consistent with the onefold-atop-site surface model. The increase in local sensitivity from using the (333) diffraction planes also enabled us to demonstrate the use of $\mathrm{x}$-ray interference fields for determining the vibrational amplitude of an adsorbate.

The possibility of using $x$-ray interference phenomena to study the bonding geometry of surface adsorbates has recently been demonstrated by using dynamical diffraction in both the Bragg ${ }^{1}$ and Laue $^{2}$ geometries. As shown in these demonstrations, an $\mathrm{X}$-ray standing-wave field can be created in the surface region of a single crystal by the interference between two coherently coupled plane waves. The antinodal planes in this interference pattern are parallel to and have the same periodicity as the acting diffraction planes. Most importantly, the phase of the standing-wave field relative to the diffraction planes can be adjusted by changing the relative phase between the two plane waves. Thus the position of an adsorbate can be measured, relative to the substrate diffraction planes, by monitoring a characteristic fluorescence signal from the adsorbate, while moving the standing-wave field continuously across the atomic planes.

The high intensity of synchrotron $x$ radiation (SXR) proved to be an essential requirement for the Laue-case measurement, since an $\mathrm{x}$-ray interferometer is needed to generate the two coherent and independently traveling wave trains that impinge on the analyzed surface. For the Bragg case, there are certain favorable measurements that can be carried out with a conventional $x$-ray source, but the necessary measuring periods of at least $10 \mathrm{~h}$ are rather long for performing systematic surface studies. With the high brilliance of SXR, such favorable measurements can be reduced down to minutes.

Until now, standing-wave measurements on surface adsorbates have used only the fundamental diffraction planes. By going to higher-order harmonics, the spatial periodicity of the excited standing wave field is reduced and therefore one is able to obtain higher-order Fourier components for the spatial distribution of the adsorbate atoms. This additional information is necessary for completely describing the adsorbate distribution, when there is more than one preferred position, and for measuring the kinetics of an adsorbate atom in terms of its vibrational amplitude. In our present investigation we have used two reflection orders for a Bragg-case $\mathrm{x}$-ray standing-wave analysis of the $\mathrm{Br} / \mathrm{Ge}(111)$ surface adsorbate system.

Prior to $\mathrm{Br}$ deposition, the symmetrically cut $\mathrm{Ge}(111)$ sample crystal was Syton polished and etched in hydrofluoric acid. The sample was transferred into a $0.05 \%$ by volume $\mathrm{Br}$ methanol solution and treated with a $\mathrm{Br}-$ methanol nonabrasive-pad polishing procedure ${ }^{3}$ followed by a thorough rinse in methanol. The sample was then kept in a drynitrogen atmosphere until the end of the measurement.
The experiment was carried out with the $x$-ray standingwave setup installed at the ROEMO instrument ${ }^{4}$ of the Hamburg Synchrotron Radiation Laboratory. Using the tunability of this instrument, the measurements were performed at an optimal incident photon energy just above the $\mathrm{Br} K$ absorption edge. This choice in energy, partially reduced $^{5}$ the overpowering influence of the $\mathrm{Ge} K \alpha$ and $K \beta$ fluorescence (at 9.9 and $11.0 \mathrm{keV}$ ) relative to the $K \alpha$ fluorescence yield (at $11.9 \mathrm{keV}$ ) from the $\mathrm{Br}$. As illustrated in Fig. 1, a double crystal monochromator was used to prepare the incident plane wave for the sample crystal. The indicated combination, of a symmetrical first crystal and an asymmetrical second crystal, reduces the angular emittance range and allows one to detune unwanted higher-order harmonics from the output beam. ${ }^{6}$ Such a detuning was used for the $\mathrm{Br} / \mathrm{Ge}(111)$ experiment. For this measurement a pair of $\mathrm{Si}(111)$ single crystals were used in the monochromator, with an asymmetrical angle of $\phi=7^{\circ}$ for the second crystal. At the selected energy of $E_{\gamma}=13.7 \mathrm{keV}$, the angular emittance range from the second crystal was $5.6 \mu \mathrm{rad}$ as

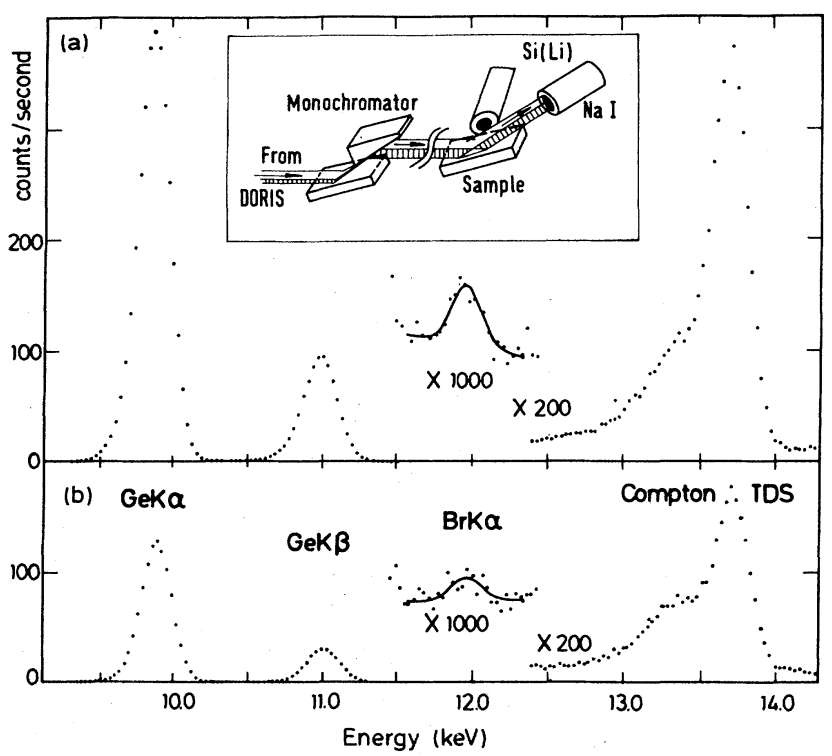

FIG. 1. $\mathrm{Br} / \mathrm{Ge}(111) \mathrm{x}$-ray spectra collected with a $\mathrm{Si}(\mathrm{Li})$ detector (shown in inset). Spectra (a) and (b) were accumulated in angular intervals 15 and 8 of Fig. 2, respectively. 
compared to $44.3 \mu \mathrm{rad}$ for the acceptance angular range of the $\mathrm{Ge}(111)$ sample crystal.

As the Ge sample crystal was repetitively rocked ${ }^{7}$ in angle $\theta$, the reflected intensity, as monitored by the $\mathrm{NaI}$ detector shown in Fig. 1, and the fluorescence spectra, were collected in a multi-spectrum-scaling mode, which subdivided the angular scan range into 16 equally spaced intervals. Spectra (a) and (b) in Fig. 1 were accumulated in angular interval 15 and 8 , respectively, during the $7.5-\mathrm{h} \mathrm{Br} / \mathrm{Ge}(111)$ experiment. The reflectivity data and $\mathrm{Br}$ fluorescence yield data for all 16 angular intervals of this scan are shown in Fig. 2.

For the $\mathrm{Br} / \mathrm{Ge}(333)$ standing-wave measurement it was necessary to eliminate the nearby (444), (555), (777), and (888) harmonics, as well as the (111) fundamental. Since asymmetrical detuning, as previously described, was not sufficient, we chose to use a pair of monochromator crystals with $d$ spacings similar to $\mathrm{Ge}(333)$, but with nonmatching $(h, k, l)$ indices. Thus we used a $\mathrm{Ge}(440)$ reflection at the first crystal and a $\mathrm{Si}(333)$ asymmetrical reflection at the second crystal. With an asymmetrical angle of $\phi=21.5^{\circ}$ and a selected energy of $E_{\gamma}=13.6 \mathrm{keV}$, the angular emittance range from the monochromator was $1.3 \mu \mathrm{rad}$ as compared to $9.2 \mu \mathrm{rad}$ for the $\mathrm{Ge}(333)$ sample. The corresponding theoretical curves and experimental data are shown in Fig. 3.

For a perfectly collimated monochromatic x-ray beam being Bragg diffracted by a symmetrically cut single crystal and polarized perpendicular to the reflection plane, the angular variation of the $K$ fluorescence yield from a specific type of atom near the surface is

$$
Y_{H}(\theta)=1+R_{H}+2 \sqrt{R_{H}} f_{c, H} \cos \left(v_{H}-2 \pi \Phi_{H}\right)
$$

The angular dependence of Eq. (1) is contained in variables $R_{H}(\theta)$ and $v_{H}(\theta)$, which correspond to the intensity and phase of the diffracted plane wave relative to the incident. The phase $v_{H}$ decreases continuously by $\pi$ radians when the crystal is advanced in angle $\theta$ through the total reflection condition. Since the photoeffect for core electrons is proportional to the $E$-field intensity at the center of the atom,

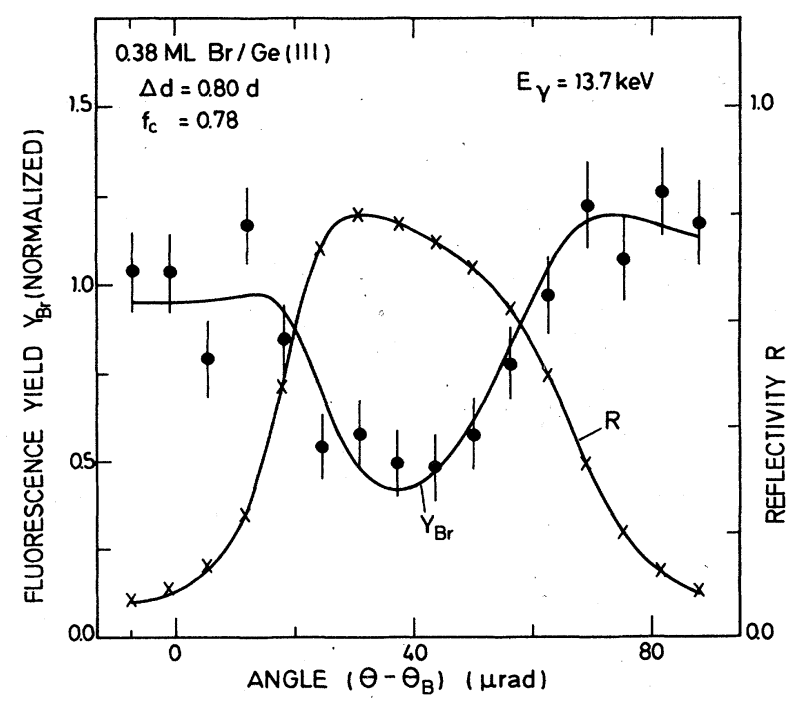

FIG. 2. Experimental data and theoretical curves for the $\mathrm{Br} K$ fluorescence yield and $\mathrm{Ge}(111)$ reflectivity vs Bragg reflection angle.

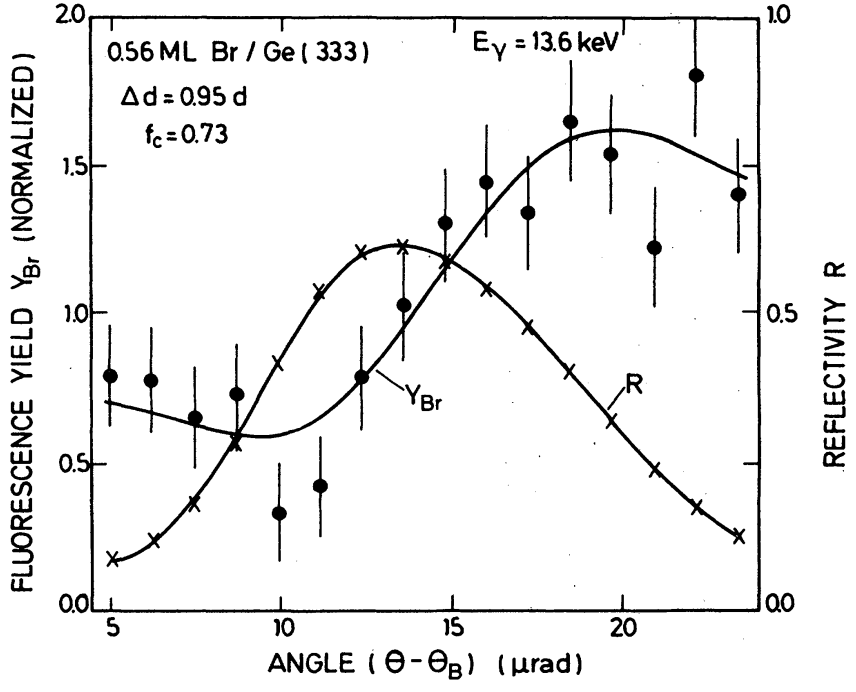

FIG. 3. Experimental data and theoretical curves for the $\mathrm{Br} K$ fluorescence yield and $\mathrm{Ge}(333)$ reflectivity vs Bragg reflection angle.

parameters $f_{c, H}$ and $\Phi_{H}$, in Eq. (1), are the amplitude and phase of the $(h, k, l)$ Fourier component $A_{H}=f_{c, H}$ $\times \exp \left(-2 \pi i \Phi_{H}\right)$ for the normalized distribution of fluorescence-selected atoms. $f_{c, H}$ and $\Phi_{H}$, which are referred to as the coherent fraction and coherent position, respectively, are determined from the $\chi^{2}$ fit of Eq. $(1)^{8}$ to the normalized fluorescence yield data as shown in Figs. 2 and 3.

From the $\mathrm{Br} / \mathrm{Ge}(111)$ data, the coherent position of the $\mathrm{Br}$ atoms with respect to the underlying perfect $\mathrm{Ge}(111)$ diffraction planes was determined to be $\Phi_{111}=0.80 \pm 0.01$. And from the $\mathrm{Br} / \mathrm{Ge}(333)$ data, the coherent position with respect to the $\mathrm{Ge}(333)$ diffraction planes was $\Phi_{333}$ $=0.95 \pm 0.03$. In Fig. 4 the $\Phi_{111}$ and $\Phi_{333}$ scales are shown along with a bulklike $\mathrm{Br} / \mathrm{Ge}(111)$ surface model. This

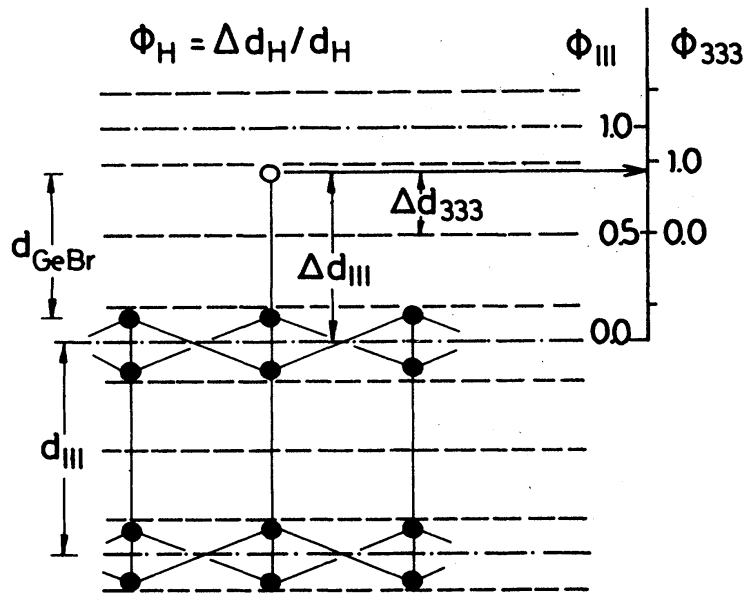

FIG. 4. $\mathrm{Br} / \mathrm{Ge}(111)$ bulklike surface model, showing $\mathrm{Br}$ atoms (O) covalently bonded to $\mathrm{Ge}(111)$ surface atoms ( $\bullet$ ) which are in ideal lattice positions, where $d_{111}=3.27 \AA$ and $d_{\mathrm{GeBr}}=2.27 \mathrm{~A}$ (Ref. 9). The (111) $(-\cdot-\cdot)$ and (333) $(---)$ diffraction planes are positioned to sense the maximum in the $\mathrm{x}$-ray scattering electron density. 
model assumes that monovalent $\mathrm{Br}$ atoms saturate the dangling bonds of Ge surface atoms and thus occupy onefoldatop positions on a locally unreconstructed and unrelaxed $\mathrm{Ge}(111)$ surface. Using bulk $\mathrm{Ge}$ and $\mathrm{GeBr}_{4}$ geometrical parameters, this model predicts that the coherent positions would be $\Phi_{111}=0.82$ and $\Phi_{333}=0.96$. This is in very good agreement (difference $<0.03 \AA$ ) with our measured values, ${ }^{10}$ whereas the threefold bridging sites on this idealized surface would place the $\mathrm{Br}$ atom several tenths of $\AA$ inward with respect to the onefold position. ${ }^{11}$ The atop-site bonding geometry is also consistent with standing-wave measurements ${ }^{2,12}$ of the chemically prepared $\mathrm{Br} / \mathrm{Si}(111)$ "real" surface and with surface extended x-ray absorption fine-structure (SEXAFS) measurements ${ }^{13,14}$ of the $\mathrm{Cl} / \mathrm{Ge}(111)$ and $\mathrm{I} / \mathrm{Ge}(111)$ "ideal" surfaces prepared in ultrahigh vacuum. Note that the inferred adsorbate bonding configuration from these measurements is identical for both surface preparations. This is attributable to the high stability of this configuration.

The relationship between the two scales shown in Fig. 4 is defined by the transformation $\Phi_{333}=3 \Phi_{111}+\frac{1}{2}(\bmod 1)$. The fact that our measured $\Phi_{111}$ and $\Phi_{333}$ values obey this transformation combined with the high coherent fractions indicates that there is only one preferred position in the [111] direction. The measured coherent fractions for the two separate experiments were $f_{c, 111}=0.78 \pm 0.03$ and $f_{c, 333}=0.73 \pm 0.06$. For such measurements, the coherent fraction can be divided into three fundamental factors as $f_{c, H}=C a_{H} D_{H}$. In this expression $C$ represents the commensurate (nonrandomly distributed) fraction of $\mathrm{Br}$ atoms, $a_{H}$ is a geometrical factor which equals unity for the case of one preferred position, and $D_{H}$ is the Debye-Waller factor for the adsorbed $\mathrm{Br}$ atom in the $\mathbf{H}$ direction. In terms of the mean square of the vibrational amplitude $\left\langle u_{H}^{2}\right\rangle$ $D_{H}=\exp \left(-2 \pi^{2}\left\langle u_{H}^{2}\right\rangle / d_{H}^{2}\right)$. Since the commensurate fraction $C$ is approximately the same for both measurements ${ }^{15}$ and since $a_{111}=a_{333}=1$ is corroborated by the high $f_{c}$ values, the $\mathrm{Br}$ vibrational amplitude can be determined from $f_{c, 111}$ and $f_{c, 333}$. From this we conclude that the chemisorbed $\mathrm{Br}$ atoms vibrate in the [111] direction with an amplitude of $(0.067 \pm 0.053) \AA$ at room temperature. In comparison, bulk $\mathrm{Ge}$ atoms at room temperature have $D_{111}=0.987$ and $D_{333}=0.889$ corresponding to $\left(\left\langle u_{111}^{2}\right\rangle\right)^{1 / 2}$ $=0.084 \AA$.

The total coverage of $\mathrm{Br}$ on the sample surface for the two different preparations was determined by comparing its off-Bragg $\mathrm{Br}$ fluorescence yield with a $\mathrm{Ge}$ sample with a known implanted dose of $\mathrm{Br}$. From this comparison the $\mathrm{Br}$ coherent coverage was $0.30 \pm 0.03$ monolayer $(\mathrm{ML})$ for the $\mathrm{Br} / \mathrm{Ge}(111)$ measurement and $0.41 \pm 0.06 \mathrm{ML}$ for the $\mathrm{Br} / \mathrm{Ge}(333)$ measurement. These values, which are obtained by multiplying the coherent fraction times the total coverage, are significantly higher than the $\mathrm{Br} / \mathrm{Si}(111)$ coherent coverages of $0.20 \mathrm{ML}{ }^{2,12}$ This increased coherent coverage can be explained by the slightly larger Ge-Ge lateral distance for two adjacent $\mathrm{Ge}$ atoms in conjunction with the filled-shell radii of two $\mathrm{Br}$ atoms. $\left(d_{110}^{\mathrm{Ge}}=4.00 \AA\right.$, $d_{110}^{\mathrm{Si}}=3.84 \AA$, and $2 r_{\mathrm{Br}^{-}}=3.90 \AA \AA^{\circ}$ ) For the bulklike $\mathrm{Br} / \mathrm{Si}(111)$ surface, adjacent $\mathrm{Br}$ atoms would slightly overlap, thus sterically forbidding the coherent coverage to exceed $\frac{1}{3}$ of a monolayler.

In conclusion, the use of multiple-order $\mathrm{x}$-ray standingwave measurements with SXR has enabled us to determine that the structure of $\mathrm{Br}$ chemisorbed onto a realistic $\mathrm{Ge}$ surface is described by a one-sited $\mathrm{Br}$ distribution function. As demonstrated, this method can also be used for measuring the vibrational amplitude of an adsorbate atom. Since $x$-ray standing-wave measurements have already been carried out in a temperature-controlled environment, ${ }^{2}$ no obstacles are foreseen for such experiments at higher or lower temperatures.
"Present address: Cornell High-Energy Synchrotron Source (CHESS) and the School of Applied and Engineering Physics, Cornell University, Ithaca, NY 14853.

${ }^{1}$ P. L. Cowan, J. A. Golovchenko, and M. F. Robbins, Phys. Rev. Lett. 44, 1680 (1980).

${ }^{2}$ G. Materlik, A. Frahm, and M. J. Bedzyk, Phys. Rev. Lett. 52, 441 (1984).

${ }^{3}$ D. E. Aspnes and A. A. Studna, Appl. Phys. Lett. 39, 316 (1981).

${ }^{4}$ G. Materlik and J. Zegenhagen, Phys. Lett. 104A, 47 (1984).

${ }^{5} \mathrm{An}$ additional reduction of the $\mathrm{Ge}$ signal relative to the $\mathrm{Br}$ was implemented by collecting the fluorescence through a $0.5-\mathrm{mm}$ aluminum attenuator.

${ }^{6} \mathrm{G}$. Materlik and V. O. Kostroun, Rev. Sci. Instrum. 51, 86 (1980).

${ }^{7}$ A. Krolzig, G. Materlik, and J. Zegenhagen, Nucl. Instrum. Methods 208, 613 (1983).

${ }^{8}$ Equation (1) is convoluted with the energy vs angle emittance window function from the monochromator. The energy width is considered since the relationship between the reflection conditions of the second and third crystal is slightly dispersive.

${ }^{9}$ Tables of Interatomic Distances and Configurations of Molecules and Ions, edited by L. E. Sutton (The Chemical Society, London, 1958).
${ }^{10}$ Note, however, that even these two Fourier components of the $\mathrm{Br}$ distribution function cannot $a$ priori serve to unambiguously describe the adsorbate geometry. This calls for additional characterization of the $\mathrm{Ge}$ top atom layer. Although such a problem can be studied with standing waves, this challenge has not yet been met successfully.

${ }^{11}$ G. B. Bachelet and M. Schluter, Phys. Rev. B 28, 2302 (1983).

12J. A. Golovchenko, J. R. Patel, D. R. Kaplan, P. L. Cowan, and M. J. Bedzyk, Phys. Rev. Lett. 49, 560 (1982).

${ }^{13}$ P. H. Citrin, J. E. Rowe, and P. Eisenberger, Phys. Rev. B 28 , 2299 (1983).

${ }^{14}$ P. H. Citrin, P. Eisenberger, and J. E. Rowe, Phys. Rev. Lett. 48, 802 (1982). To our present knowledge there are no reported SEXAFS measurements for the $\mathrm{Br} / \mathrm{Si}(111)$ or $\mathrm{Br} / \mathrm{Ge}(111)$ surfaces.

${ }^{15}$ From the reproducibility of the chemical preparation process for $\mathrm{Br} / \mathrm{Si}(111)$ and $\mathrm{Br} / \mathrm{Ge}(111)$, we estimate the total error of $f_{c, 111}$ to be \pm 0.05 .

${ }^{16}$ C. Kittel, Introduction to Solid State Physics, 4th ed. (Wiley, New York, 1971). 